\title{
Viabilidade de implantação de ureias revestidas no cultivo de milho para Estado de São Paulo
}

\author{
Viability of coated urea introduction in maize in São Paulo State
}

\author{
Edson Pereira da Mota $^{1 * 1} ;$ Fernanda Latanze Mendes², Ricardo Shirota $^{3}$ \\ 'ESALQ/USP - Doutorando em Ciências (Solos e Nutrição de Plantas) - Av. Pádua Dias 11, Departamento de Ciência \\ do Solos - CEP 13418-900 - Piracicaba (SP), Brasil \\ 2IPECEGE, Doutora em Ciências (Solos e Nutrição de Plantas) - Rua Alexandre Herculano 120, sala T4, Vila Monteiro - \\ CEP 13418-445 - Piracicaba (SP), Brasil \\ ${ }^{3}$ ESALQ/USP - Prof. Dr. Departamento de Economia, Administração e Sociologia - Av. Pádua Dias 11 - CEP 13418- \\ 900 - Piracicaba (SP), Brasil
}

\section{Resumo}

O mercado de fertilizantes vem passando por intensa fase de desenvolvimento envolvendo pesquisas relacionadas a novas tecnologias aplicadas aos fertilizantes. Uma nova gama de insumos, como os fertilizantes recobertos, é disponibilizada à compra e uso pelos produtores mostrando a fase de intensificação e tecnificação ocorrida, hoje, na agricultura. Essa variedade abre ao produtor novos caminhos e estratégias de manejos, mas, também, levanta a questão da viabilidade de aplicação destes produtos em substituição aos insumos convencionais dado o inevitável aumento de preço resultante da agregação de tecnologia à forma tradicional do insumo. Assim o recobrimento da ureia é estrategicamente recomendado podendo resultar em ganhos pela diminuição de perdas e, por ser o fertilizante mais concentrado em $\mathrm{N}$ com menor custo por unidade de nutriente. Através da metodologia da orçamentação parcial, calculou-se as alterações nos custos de produção e receita econômica obtida da substituição da ureia comum por quatro fertilizantes recobertos (UA, UB, UC e UD) com o objetivo da verificação da viabilidade desta troca no cultivo agrícola do milho de $1^{\underline{a}}$ safra. Quatro diferentes cenários foram simulados considerando produtividade esperada de $8,5 \mathrm{t} / \mathrm{ha}$, constatou-se que, para os dois primeiros, os fertilizantes recobertos foram inviáveis a substituição da ureia convencional e, para os dois últimos, apenas o fertilizante UA foi viável à substituição. Verificou, também, que há a necessidade de aumento de produtividade para que haja o nivelamento da ureia convencional com as recobertas, ponto a partir do qual a substituição do sistema convencional pelos produtos recobertos torna-se viável.

Palavras-chave: custo de produção, fertilizante, nitrogênio, perdas

\section{Abstract}

The fertilizer market has been experiencing intense development stage involving researches related to new technologies applied to fertilizers. A new line of inputs such as coated fertilizers is available to purchase and use by farmers, showing the intensification and technification stage occurring today in agriculture. These possibilities opens up to farmers new ways and managements strategies, but also raises the question of the viability of these products in substitution of inputs already used due to the inevitable price increase result of the technology aggregation to the input in its traditional form. So coated urea is strategically recommended and may result in gains due to loss decrease and because urea is the most concentrated $\mathrm{N}$ fertilizer with lower cost per unit of nutrient. Through the methodology of partial budgeting, it was calculated the changes in production costs and economic revenues obtained from the replacement of common urea for four coated fertilizers (UA, UB, UC and UD) with the aim of verifying the feasibility of this exchange in the agricultural cultivation of maize planted in summer. Four different scenarios were simulated considering expected yield of $8.5 \mathrm{t} / \mathrm{h}$. The first two were not feasible to

\footnotetext{
* Autor correspondente <edson_mota@agronomo.eng.br> Enviado: 03 ago. 2014

Aprovado: 29 nov. 2014
} 
replace the conventional urea and the last two, only the fertilizer UA was feasible to replace. It was verified that there is the need to increase productivity so that there would be the leveling of conventional urea and coated urea from which the replacement of the conventional system by the covered products becomes feasible.

Key words: production costs, fertilizer, nitrogen, losses

\section{Introdução}

No Brasil, o milho ocupa o segundo lugar em área plantada, estimada em 15,5 milhões de hectares ficando atrás apenas da soja, com 28 milhões de hectares. A produção total de milho (primeira e segunda safras) ultrapassou os 80 milhões de toneladas. Entretanto, as médias de produtividade brasileira estão situadas em 5,5 t/ha, valor que possui grande potencial de aumento com a tecnificação do campo e melhores produtos e orientações aos produtores (CONAB, 2014).

No Estado de São Paulo, a área total, tende a diminuir linearmente passando de 1,16 milhão de hectares no ano de 2000 para 0,9 milhão de hectares em 2013 (CONAB, 2014). Para a manutenção e contínuos aumentos da produção e produtividade desta cultura, são necessários cuidados no momento da elaboração do programa de adubação de forma bem estruturada que atenda a todas as necessidades nutricionais do milho, já que a planta é exigente em nutrientes e fertilidade do solo (Raij, 1997; Fancelli, 2009).

$\mathrm{Na}$ adubação, o nitrogênio $(\mathrm{N})$ destaca-se por ser o elemento de maior extração pela cultura atingindo até $80 \%$ de extração nutricional (Marchner, 1995; Fancelli; Tsumanuma, 2007). Em termos gerais, recomenda-se aplicar entre 10 e $50 \mathrm{~kg} / \mathrm{ha}$ de $\mathrm{N}$ na semeadura, considerando o rendimento esperado, salientando-se que aplicações superiores a estas doses podem acarretar problemas de salinização do solo e/ou alcalinização da rizosfera, dependendo da fonte a ser utilizada. A adubação de cobertura é recomendada entre os estádios V4 e V8 (emissão da $4^{a}$ a $8^{\text {a }}$ folhas) com até $150 \mathrm{~kg} /$ ha de N (Raij, 1997; Fancelli, 2009; Rodrigues; Guadagnin; Porto, 2009).

Dentre as fontes disponíveis, tem-se os fertilizantes com tecnologia agregada que constituem um grupo de fertilizantes convencionais onde há a aplicação de novas tecnologias de caráter físico, químico ou biológico na busca da maior eficiência de uso das fontes e na redução de possíveis perdas agronômicas, como volatilização e lixiviação evitando a contaminação de águas subterrâneas e superficiais (Shaviv, 2001). Através do uso desta tecnologia, pode-se possibilitar a liberação do $\mathrm{N}$ em sincronia com o crescimento da planta providenciando $\mathrm{N}$ suficiente em uma única aplicação e ainda conservar muito baixas as concentrações deste nutriente nos solos durante 0 crescimento da cultura (Peoples; Freney; Mosier, 1995). 
Tais fertilizantes tecnológicos enquadram a libertação controlada e/ou lenta, estes retardam a disponibilidade inicial dos nutrientes ou incrementam a sua disponibilidade no tempo através de diferentes mecanismos. Há os produtos de degradação microbiana e inibidores, como ureia-formaldeído e outras ureia-aldeídos e NBPT que, comercialmente designam-se como fertilizantes de libertação lenta ("slowrelease fertilizer") (Trenkel, 1997, 2010).

Os fertilizantes revestidos ou encapsulados por películas de enxofre ou polímero de ou polímeros de natureza diversa são designados de fertilizantes de libertação controlada ("controlled release fertilizer") (TrenkeL, 1997, 2010) promovem a liberação do $\mathrm{N}$ de forma mais lenta (Vitti; Heirinchs, 2007) devido à barreira física contra a exposição do nutriente (Blaylock, 2007). Esta barreira evita o contato da ureia com o solo e, por conseguinte, a hidrólise da mesma e as chances de volatilização são reduzidas.

O processo de encapsulação influi diretamente no mecanismo e intensidade do processo de liberação. A espessura, a porosidade e a natureza química da resina de recobrimento, a quantidade de microfissuras superficiais e o tamanho do grânulo de fertilizante contribuem para determinar a curva de liberação de nutrientes ao longo do tempo (Trenkel, 1997). Considerando estes fatores, a análise de viabilidade de aplicação dessas novas tecnologias para que não somente tenhamos os benefícios voltados ao cultivo agrícola, mas também voltado ao sistema de produção como um todo, ou seja, a escolha do gerenciamento mais adequado e rentável na atividade, pelo produtor, torna-se fundamental. Dessa forma, fecha-se o ciclo: desenvolvimento de produto - aplicação - retorno econômico; e, assim, tem-se a informação completa das mudanças que podem ocorrer na cadeia produtiva agrícola.

Em vista desses parâmetros, o objetivo foi estudar a viabilidade de implantação de ureias revestidas com polímeros em diferentes cenários, na cultura do milho de primeira safra para o Estado de São Paulo, em substituição aos insumos convencionais (sem tecnologia agregada).

\section{Materiais e Métodos}

Fontes de dados

Os dados coletados a respeito do custo de produção do cultivo de milho, custo de obtenção da ureia e produção/produtividade do milho (com e sem ureia revestida) foram obtidos via revisão de literatura disponível como anuários agrícolas, livros, 
instituições governamentais (como CONAB, ANDA e IEA), artigos científicos e resumos de congressos.

Dada a dificuldade de obtenção de dados relativos ao preço do fertilizante revestido, estes foram coletados via informação pessoal e consultas às próprias empresas fabricantes do insumo e seus representantes.

\section{Análises dos dados}

Foram levantados dados de produção, produtividade, preço e custos de produção do milho de 1a safra, para o Estado de São Paulo, no período de 2005 a 2013 obtidos de levantamentos realizados pela Companhia Nacional de Abastecimento (CONAB) e pelo Agrianual (AGRAFNP, 2014).

Para a análise dos cenários aplicou a metodologia da orçamentação, pela qual a identificação do capital adicionado ou subtraído de um processo, e sua consequente modificação na receita gerada, verificou-se por comparação o melhor saldo gerado. Porém, o método se limita em apenas permitir conhecer o aumento ou diminuição na renda pela modificação do processo a ser adotado (Hoffmann et. al, 1992).

Os valores da ureia e as recobertas também foram coletados. Para a ureia comum, utilizaram-se os levantamentos realizados pela CONAB e, para os produtos recobertos, dado a dificuldade da obtenção de informações publicadas a respeito de preço, realizaram-se consultas pessoais diretamente aos representantes das empresas produtoras destes produtos, estes foram denominados UA, UB, UC e UD (Tabela 1).

Tabela 1. Preço da ureia e dos produtos recobertos

\begin{tabular}{cc}
\hline Fertilizantes & Preço \\
\hline & $\mathrm{R} \$ / \mathrm{t}$ \\
Ureia & $1.466,00$ \\
UA & $1.790,00$ \\
UB & $2.100,00$ \\
UC & $4.000,00$ \\
UD & $9.000,00$
\end{tabular}

Fonte: CONAB (2014); Comunicação pessoal

Os dados foram organizados e, através da análise dos custos de produção identificou a parte dos custos atribuídos ao uso dos fertilizantes e, dentro destes, os dos nitrogenados, especificamente o uso da ureia (Tabela 2). 
A identificação da participação da ureia nos custos permitiu a substituição desta pelos produtos recobertos (área destacada da Tabela 2), assim simulações utilizando a ureia sem recobrimento e, os 4 produtos recobertos com diferentes tecnologias e diferentes preços de venda, foram traçados e registrou-se os custos de produção totais.

Quatro diferentes cenários foram elaborados considerando possibilidades de modificação no sistema de produção, para todos os cenários considerou-se a produtividade de 8,5 t/ha. A descrição dos cenários é mostrada abaixo:

- Cenário 1: análise dos custos de produção da ureia e substituição desta pelos produtos recobertos, foi considerada dose de $180 \mathrm{~kg} / \mathrm{ha}$ de $\mathrm{N}$, aplicada em 2 operações (plantio - 50 kg/ha; cobertura $130 \mathrm{~kg} / \mathrm{ha}$ );

- Cenário 2: análise dos custos de produção das ureias recobertas considerando dose de $180 \mathrm{~kg} / \mathrm{ha}$ aplicada em apenas 1 operação (plantio - $180 \mathrm{~kg} / \mathrm{ha}$ ), para ureia sem recobrimento foi mantida a realização de 2 operações de adubação (plantio - $50 \mathrm{~kg} / \mathrm{ha}$; cobertura $130 \mathrm{~kg} / \mathrm{ha}$ );

- Cenário 3: análise dos custos de produção da ureia e dos produtos recobertos. Para a ureia foi considerado dose de $180 \mathrm{~kg} / \mathrm{ha}$ de $\mathrm{N}$ aplicada em 2 operações (plantio - $50 \mathrm{~kg} / \mathrm{ha}$; cobertura $130 \mathrm{~kg} / \mathrm{ha}$ ), para os produtos recobertos, foi considerada redução de $20 \%$ da dose utilizada para ureia sem recobrimento (144 kg/ha), aplicada em 2 operações (plantio 40 kg/ha; cobertura 104 kg/ha);

- Cenário 4: análise dos custos de produção das ureias recobertas. Foi considerada redução de $20 \%$ da dose utilizada para ureia sem recobrimento (144 kg/ha), aplicada em 1 operação (plantio 144 kg/ha);

A análise da viabilidade da adoção da ureia, ou a substituição desta pelos produtos recobertos, realizada por meio da metodologia de orçamentação parcial substituindo, nos custos de produção, as alternativas propostas (cenários) e verificando os possíveis aumentos de custo e/ou de receitas e comparando os resultados econômicos obtidos (Tabela 3). 
Tabela 2. Custo de produção do milho para produtividade esperada de 8,5 t/ha

\begin{tabular}{|c|c|c|c|c|c|}
\hline & Descrição & Especificação & V.U & Qtde. & Valor \\
\hline $\mathbf{A}$ & Operações & & & & \\
\hline \multirow[t]{2}{*}{ A.1 } & Conservação do solo & & & & \\
\hline & Manutenção de carreadores & HM Tp $4 \times 4125 c v$ + plaina traseira & 90,04 & 0,10 & 9,00 \\
\hline \multirow[t]{2}{*}{ A2 } & Preparo do solo & & & & \\
\hline & Calagem & HM Tp 4×2 65cv + distrb. Calcário 2,3m3 & 71,07 & 0,10 & 7,11 \\
\hline \multirow[t]{4}{*}{ A.3 } & $\begin{array}{l}\text { Plantio } \\
\text { Plantio }\end{array}$ & IM Tp 4x4 125cv + plantadora PD 10 linha & 106,51 & 1,00 & 106,51 \\
\hline & Tratamento de sementes & Misturador de sementes & 15,68 & 0,10 & 1,57 \\
\hline & Serviço braçal & $\mathrm{HH}$ trablhador braçal & 5,30 & 0,60 & 3,18 \\
\hline & Transportes internos & HM Tp $4 \times 265 c v$ + carreta 4 toneladas & 81,11 & 0,10 & 8,11 \\
\hline \multirow[t]{5}{*}{ A4 } & Tratos culturais & & & & \\
\hline & Adubação de cobertura & HM Tp 4x2 65cv + cultivador adubador & 77,00 & 0,40 & 30,80 \\
\hline & Aplicação de Defensivos & Autopropelido & 184,93 & 0,25 & 46,23 \\
\hline & Combate a formigas/cupins & $\mathrm{HH}$ trablhador braçal & 5,30 & 0,15 & 0,80 \\
\hline & $\begin{array}{l}\text { Transportes internos } \\
\text { Colheita }\end{array}$ & HM Tp $4 \times 265 c v$ + carreta 4 toneladas & 81,11 & 0,10 & 8,11 \\
\hline \multirow{2}{*}{ A.5 } & Colheita mecânica & HM Colhedora & 218,60 & 0,60 & 131,16 \\
\hline & Transportes internos & HM Tp $65 \mathrm{cv} .+$ carreta 4 toneladas & 81,11 & 0,15 & 12,17 \\
\hline \multicolumn{2}{|c|}{ Subtotal A } & \multicolumn{4}{|r|}{364,75} \\
\hline B & Insumos & & & & \\
\hline \multirow[t]{4}{*}{ B.1 } & $\begin{array}{c}\text { Fertilizantes/Corretivos } \\
\text { Calcário }\end{array}$ & $\mathrm{R} \$ /$ tonelada & 100,00 & 0,35 & 35,00 \\
\hline & Ureia & $\mathrm{R} \$ /$ tonelada & 1466,00 & 0,40 & 586,40 \\
\hline & Cloreto de potássio & $\mathrm{R} \$$ /tonelada & 1215,00 & 0,10 & 121,50 \\
\hline & 08-28-12 + Micronutrientes & $\mathrm{R} \$ /$ tonelada & 1257,00 & 0,40 & 502,80 \\
\hline \multirow{2}{*}{ B.2 } & Sementes/Mat. Plantio & & & & \\
\hline & Sementes & $\mathrm{R} \$ / 60.000$ & 275,00 & 1,20 & 330,00 \\
\hline \multirow[t]{6}{*}{ B.3 } & Defensivos agrícolas & & & & \\
\hline & Formicidas & $\mathrm{R} \$ / \mathrm{kg}$ & 12,00 & 0,50 & 6,00 \\
\hline & Herbicidas & $\mathrm{R} \$ /$ litro & 16,92 & 12,00 & 203,04 \\
\hline & Inseticidas & $\mathrm{R} \$ /$ litro & 158,48 & 0,50 & 79,24 \\
\hline & Fungicida & $\mathrm{R} \$$ /litro & 86,00 & 0,75 & 64,50 \\
\hline & Outros & $\mathrm{R} \$$ /litro & 12,00 & 1,50 & 18,00 \\
\hline \multicolumn{2}{|c|}{ Subtotal B } & \multicolumn{4}{|r|}{1946,48} \\
\hline \multirow[t]{8}{*}{ C } & Administração & & & & \\
\hline & M.O Administrativa & $(\mathrm{R} \$ / \mathrm{ha})$ & 39,55 & 1,00 & 39,55 \\
\hline & Assistência Técnica & $(\mathrm{R} \$ / \mathrm{ha})$ & 22,60 & 1,00 & 22,60 \\
\hline & Contábil/Escritório & $(\mathrm{R} \$ / \mathrm{ha})$ & 9,04 & 1,00 & 9,04 \\
\hline & Luz/Telefone & $(\mathrm{R} \$ / \mathrm{ha})$ & 11,30 & 1,00 & 11,30 \\
\hline & Conserv./Deprec./Benf. & $(\mathrm{R} \$ / \mathrm{ha})$ & 12,60 & 1,00 & 12,60 \\
\hline & Viagens & $(\mathrm{R} \$ / \mathrm{ha})$ & 5,75 & 1,00 & 5,75 \\
\hline & Impostos/Taxas & $\%$ Receita & 2,30 & 1,00 & 68,43 \\
\hline \multicolumn{2}{|c|}{ Subtotal C } & \multicolumn{4}{|r|}{169,27} \\
\hline \multirow[t]{5}{*}{ D } & Pós colheita & & & & \\
\hline & Transporte até armazenagem & $\mathrm{R} \$ /$ tonelada & 9,00 & 8,50 & 76,50 \\
\hline & Recebimento/Limpeza/Secagem & $\mathrm{R} \$ /$ tonelada & 30,83 & 8,50 & 262,06 \\
\hline & Armazenagem & $\mathrm{R} \$ /$ tonelada & 8,33 & 8,50 & 70,81 \\
\hline & Taxa administrativa & $\mathrm{R} \$ /$ tonelada & 3,92 & 8,50 & 33,32 \\
\hline \multicolumn{2}{|c|}{ Subtotal D } & \multicolumn{4}{|r|}{442,68} \\
\hline \multirow{4}{*}{\multicolumn{2}{|c|}{$\begin{array}{l}\text { Custo total (R\$/ha) } \\
\text { Receita }(\mathrm{R} \$ / \mathrm{ha}) \\
\text { Preço médio }(\mathrm{R} \$ / \mathrm{sc} 60 \mathrm{~kg}) \\
\text { Resultado (R\$/ha) }\end{array}$}} & & & & 2923,18 \\
\hline & & & & & 3785,33 \\
\hline & & & & & 26,72 \\
\hline & & & & & 862,16 \\
\hline
\end{tabular}

Fonte: adaptado de AGRIANUAL (2014) 
Tabela 3. Cenários de uso da ureia e produtos recobertos

\begin{tabular}{|c|c|c|c|}
\hline Cenário 1 & Preço fertilizantes & Custo de Prod.Total & Receita \\
\hline Insumo & & $\mathrm{R} \$ /$ ha & \\
\hline Ureia & 1466,00 & 2923,18 & 3785,33 \\
\hline UA & 1790,00 & 3052,78 & 3785,33 \\
\hline UB & 2100,00 & 3176,78 & 3785,33 \\
\hline UC & 4000,00 & 3936,78 & 3785,33 \\
\hline UD & 9000,00 & 5936,78 & 3785,33 \\
\hline Cenário 2 & Preço fertilizantes & Custo de Prod.Total & Receita \\
\hline Insumo & & $\mathbf{R} \$ / \mathbf{h a}$ & \\
\hline Ureia & 1466,00 & 2923,18 & 3785,33 \\
\hline UA & 1790,00 & 3021,98 & 3785,33 \\
\hline UB & 2100,00 & 3145,98 & 3785,33 \\
\hline UC & 4000,00 & 3905,98 & 3785,33 \\
\hline UD & 9000,00 & 5905,98 & 3785,33 \\
\hline Cenário 3 & Preço fertilizantes & Custo de Prod.Total & Receita \\
\hline Insumo & & $\mathbf{R} \$ /$ ha & \\
\hline Ureia & 1466,00 & 2923,18 & 3785,33 \\
\hline UA & 1790,00 & 2909,58 & 3785,33 \\
\hline UB & 2100,00 & 3008,78 & 3785,33 \\
\hline UC & 4000,00 & 3616,78 & 3785,33 \\
\hline UD & 9000,00 & 5216,78 & 3785,33 \\
\hline Cenário 4 & Preço fertilizantes & Custo de Prod.Total & Receita \\
\hline Insumo & & $\mathbf{R} \$ /$ ha & \\
\hline Ureia & 1466,00 & 2923,18 & 3785,33 \\
\hline UA & 1790,00 & 2878,78 & 3785,33 \\
\hline UB & 2100,00 & 2977,98 & 3785,33 \\
\hline UC & 4000,00 & 3585,98 & 3785,33 \\
\hline UD & 9000,00 & 5185,98 & 3785,33 \\
\hline
\end{tabular}

Ressalta-se que as receitas obtidas foram calculadas com base na produtividade esperada (8,5 t/ha), convertida em sacas de $60 \mathrm{~kg}$ e multiplicada pelo valor de venda $(\mathrm{R} \$ 26,72)$.

$\mathrm{Na}$ ocorrência da substituição da ureia comum pelos produtos recobertos não se apresentar viável. Calculou-se a necessidade de aumento de produção, tanto em sc/ha quanto $\mathrm{em} \mathrm{kg} / \mathrm{ha}$, que o uso destes produtos necessitaria para a receita se igualar aos custos, ou seja, o ponto de nivelamento necessário a partir do qual o uso dos fertilizantes com tecnologia agregada se tornasse viável, como apresentado nas equações 1 e 2.

$$
N A P(s c / h a)=\frac{A C S}{P V}
$$




$$
N A P(k g / h a)=\frac{A C S}{P V} \times 60
$$

Onde, NAP: Necessidade de Aumento de Produtividade; ACS: Aumento de Custo devido Substituição do fertilizante; PV: Preço de Venda do produto (sc $60 \mathrm{~kg}$ milho).

A realização destes cálculos indica, em caso de resultado econômico negativo, qual o mínimo de elevação de produtividade a tecnologia deve produzir para que os gastos com sua aplicação nos fertilizantes sejam cobertos sem prejuízo ao produtor.

\section{Resultados e Discussão}

Análise dos cenários

A análise do primeiro cenário simulado (dose de $180 \mathrm{~kg} / \mathrm{ha}$ aplicada em duas operações de adubação), por manter constantes os valores de produtividade, dose e operações realizadas, mostra que a utilização da ureia sem recobrimento, via metodologia de orçamentação parcial, é a alternativa mais viável a ser adotada (Tabela 4). Tal fato ocorre pela simples comparação dos preços da ureia frente aos produtos recobertos, dado os maiores preços resultantes da aplicação de tecnologia ao fertilizante convencional, o aumento nos custos pelo uso das ureias recobertas onera os custos de produção de $R \$ 129,60$ para o produto UA, chegando a até $R \$ 3.013,60$ para o produto UD, ressaltando-se que esse aumento é calculado considerando os custos de produção utilizando apenas ureia não recoberta como base (valor 0) (Figura 1a). Ressalta-se, também, que os aumentos nos custos de produção são iguais a redução nos resultados econômicos frente a ureia, valor base adotado.

Os resultados econômicos deste cenário mostram que o maior valor obtido, através do uso do fertilizante na forma convencional, foi calculado em $R \$ 862,16$ por hectare cultivado com milho de primeira safra e é o mais rentável na atividade sendo seguido por UA com $R \$ 732,56$, UB com $R \$ 608,56$ e, para os produtos UC e UD, temos resultado econômico negativo, ou seja, a adoção destas alternativas resulta em resultado de $R \$ 151,44$ e $R \$ 2151,44$ negativos por hectare, respectivamente. Dessa forma, a aplicação de tecnologia, para este cenário não é alternativa viável a substituição do método convencional aplicado no campo (Figura 1a). 
Tabela 4. Resultados dos cenários simulados pela metodologia de orçamentação parcial

Cenário 1 Preço fert. Custo de Prod.Total Receita Resultado Aumento de custo Necessidade de aumento de produt.

\begin{tabular}{|c|c|c|c|c|c|c|c|}
\hline Insumo & $\ldots$ & 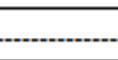 & $R \$ / h a$ & 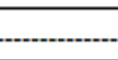 & ב-n & sc/ha & $\mathrm{kg} / \mathrm{ha}$ \\
\hline Ureia & 1466,00 & 2923,18 & 3785,33 & 862,16 & 0,00 & 0,0 & 0 \\
\hline UA & 1790,00 & 3052,78 & 3785,33 & 732,56 & 129,60 & 4,9 & 291,0 \\
\hline UB & 2100,00 & 3176,78 & 3785,33 & 608,56 & 253,60 & 9,5 & 569,5 \\
\hline UC & 4000,00 & 3936,78 & 3785,33 & $-151,44$ & 1013,60 & 37,9 & 2276,0 \\
\hline UD & 9000,00 & 5936,78 & 3785,33 & $-2151,44$ & 3013,60 & 112.8 & 6767.1 \\
\hline
\end{tabular}

Cenário 2 Preço fert. Custo de Prod. Total Receita Resultado Aumento de custo Necessidade de aumento de produt.

\begin{tabular}{|c|c|c|c|c|c|c|c|}
\hline Insumo & 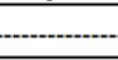 & & $\mathrm{RS} / \mathrm{ha}$ & 5 & 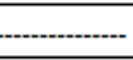 & sc/ha & $\mathrm{kg} / \mathrm{ha}$ \\
\hline Ureia & 1466,00 & 2923,18 & 3785,33 & 862,16 & 0,00 & 0,0 & 0 \\
\hline UA & 1790,00 & 3021,98 & 3785,33 & 763,36 & 98,80 & 3,7 & 221,9 \\
\hline UB & 2100,00 & 3145,98 & 3785,33 & 639,36 & 222,80 & 8,3 & 500,3 \\
\hline UC & 4000,00 & 3905,98 & 3785,33 & $-120,64$ & 982,80 & 36,8 & 2206,9 \\
\hline UD & 9000,00 & 5905,98 & 3785,33 & $-2120,64$ & 2982,80 & 111,6 & 6697,9 \\
\hline
\end{tabular}

Cenário 3 Preço fert. Custo de Prod. Total Receita Resultado Aumento de custo Necessidade de aumento de produt.

\begin{tabular}{|c|c|c|c|c|c|c|c|}
\hline Insumo & 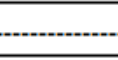 & & $\mathrm{RS} / \mathrm{ha}$ & 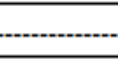 & ב-1- & sc/ha & $\mathrm{kg} / \mathrm{ha}$ \\
\hline Ureia & 1466,00 & 2923,18 & 3785,33 & 862,16 & 0,00 & 0,0 & 0 \\
\hline UA & 1790,00 & 2909,58 & 3785,33 & 875,76 & $-13,60$ & $-0,5$ & $-30,5$ \\
\hline UB & 2100,00 & 3008,78 & 3785,33 & 776,56 & 85,60 & 3,2 & 192,2 \\
\hline UC & 4000,00 & 3616,78 & 3785,33 & 168,56 & 693,60 & 26,0 & 1557,5 \\
\hline UD & 9000,00 & 5216,78 & 3785,33 & $-1431,44$ & 2293,60 & 85,8 & 5150,3 \\
\hline
\end{tabular}

Cenário 4 Preço fert. Custo de Prod. Total Receita Resultado Aumento de custo Necessidade de aumento de produt.

\begin{tabular}{|c|c|c|c|c|c|c|c|}
\hline \multirow{2}{*}{$\begin{array}{l}\text { Insumo } \\
\text { Ureia }\end{array}$} & \multicolumn{5}{|c|}{ 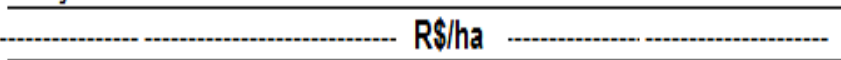 } & \multirow{2}{*}{$\begin{array}{c}\text { sc/ha } \\
0,0\end{array}$} & \multirow{2}{*}{$\begin{array}{c}\mathrm{kg} / \mathrm{ha} \\
0\end{array}$} \\
\hline & 1466,00 & 2923,18 & 3785,33 & 862,16 & 0,00 & & \\
\hline UA & 1790,00 & 2878,78 & 3785,33 & 906,56 & $-44,40$ & $-1,7$ & $-99,7$ \\
\hline UB & 2100,00 & 2977,98 & 3785,33 & 807,36 & 54,80 & 2,1 & 123,1 \\
\hline UC & 4000,00 & 3585,98 & 3785,33 & 199,36 & 662,80 & 24,8 & 1488,3 \\
\hline UD & 9000,00 & 5185,98 & 3785,33 & $-1400,64$ & 2262,80 & 84,7 & 5081,1 \\
\hline
\end{tabular}

A análise do segundo cenário (dose de $180 \mathrm{~kg} / \mathrm{ha}$ aplicada em apenas uma operação de adubação) permite verificar que, mesmo com a eliminação dos custos com a adubação de cobertura, as diminuições resultantes nos custos de produção utilizando os produtos recobertos não são suficientes para estas alternativas se tornarem viáveis. Os aumentos nos custos utilizando as ureias recobertas são de $R \$ 98,80$ para UA, $R \$$ 222,80 para UB e, para UC e UD temos $R \$ 982,80$ e $R \$ 2982,80$, respectivamente (Tabela 4). Estes aumentos não são cobertos de forma a resultar resultados econômicos satisfatórios e, desta forma, os fertilizantes recobertos aplicados via adubação única não são alternativas viáveis à adoção (Figura 1b). 
Pode-se considerar que, para este cenário, quanto maiores os custos com a operação da adubação de cobertura, maior será a diminuição dos custos de produção total dos fertilizantes recobertos. Mantendo-se a condição ceteris paribus, os produtos recobertos se tornariam viáveis apenas no caso da operação de cobertura para ureia sem recobrimento superar o valor do aumento causado pelos maiores preços dos produtos com tecnologia agregada.
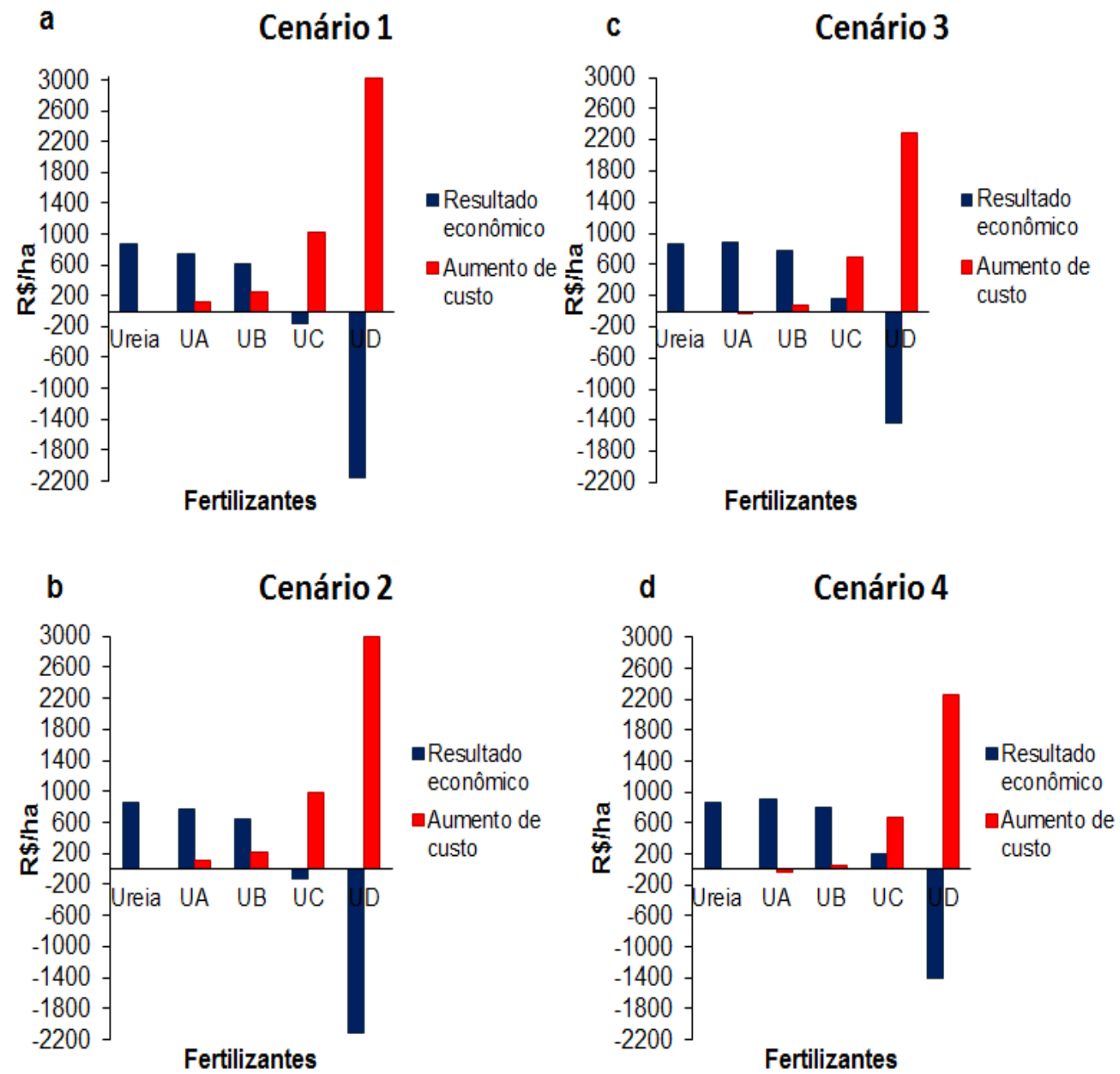

Figura 1. Gráfico de aumento de custo x retornos econômicos resultantes dos cenários simulados

Os dados apresentados no cenário 3 (redução de $20 \%$ na dose para os produtos recobertos aplicados em 2 operações de adubação frente as operações convencionais com ureia sem recobrimento) mostraram que para o produto UA, este apresentou como a alternativa mais viável à adoção por apresentar redução nos custos de produção totais, ressaltando-se que as operações com ureia sem recobrimento foram 
consideradas como base (valor 0), e elevação no retorno econômico de mesma magnitude (Figura 1c). A redução nos custos utilizando UA, e consequente aumento de produção, foi de $R \$ 13,60$ por hectare cultivado (Tabela 4).

As demais alternativas, em ordem de viabilidade, foram a ureia sem recobrimento seguida do produto UB, com seu aumento de custos e retornos econômicos de $R \$ 85,60$ e $R \$ 807,36$, respectivamente. Neste cenário verificou que o produto UC obteve resultado positivo de $\mathrm{R} \$ 168,56$, porém, muito abaixo do uso da ureia sem tecnologia agregada e, para o produto UD devido seu alto preço, que resultou em alto aumento de custos, estes apresentaram retornos econômicos negativos atingindo $\mathrm{R} \$ 1431,44$ por hectare.

O cenário 4 (redução de $20 \%$ da dose aplicada em apenas uma operação de adubação, frente a dose total de ureia aplicada em 2 operações) apresentou o produto UA como alternativa mais viável, valor já esperado após realização da simulação do cenário 3, pois além da redução de dose, o cenário 4 eliminou os custos com a aplicação do fertilizante em cobertura. Os ganhos frente à ureia sem recobrimento, nos resultados econômicos, atingiram $R \$ 44,40$, valor referente à diminuição causada nos custos de produção (Tabela 4).

As demais alternativas, em ordem de viabilidade, para o cenário 4 foram a ureia sem recobrimento (valor base), o produto UB com resultado de $\mathrm{R} \$ 54,80$ a menos que a ureia sem tecnologia agregada e, UC e UD com valores de aumento de custos e redução de retorno econômico de $\mathrm{R} \$ 662,80$ e $\mathrm{R} \$ 2.262,80$, respectivamente (Figura 1d). Ressalta-se que, assim como cenário 2 os produtos recobertos seriam viáveis apenas quando do custo da operação de adubação e cobertura for superior aumento de custo causado pelos preços mais elevados dos fertilizantes com tecnologia agregada (na condição ceteris paribus).

\section{Comparação de cenários}

Ao realizar a junção entre os orçamentos parciais dos diferentes cenários simulados e, tendo como base os valores da ureia sem recobrimento na dose de 180 $\mathrm{kg} / \mathrm{ha}$ de $\mathrm{N}$ aplicada em duas operações de adubação (plantio e cobertura), que se repete em todos os cenários, foi possível, através do uso de gráficos, traçar linhas de base que auxiliem na visualização dos resultados econômicos (Figura 2 e 3). Essas linhas se situam, para os resultados econômicos, no valor de $R \$ 862,16$ e, para os aumentos de produção, como adotou-se a ureia como base, a linha coincide ao eixo das abscissas. 
Os resultados econômicos da ureia (constantes nas quatro simulações) foram superados apenas pelo produto UA e, apenas nos cenários 3 e 4 , resultado que consequentemente se repetiu nos aumentos de custos dada a dependência destes valores (Figura 2 e 3 ).

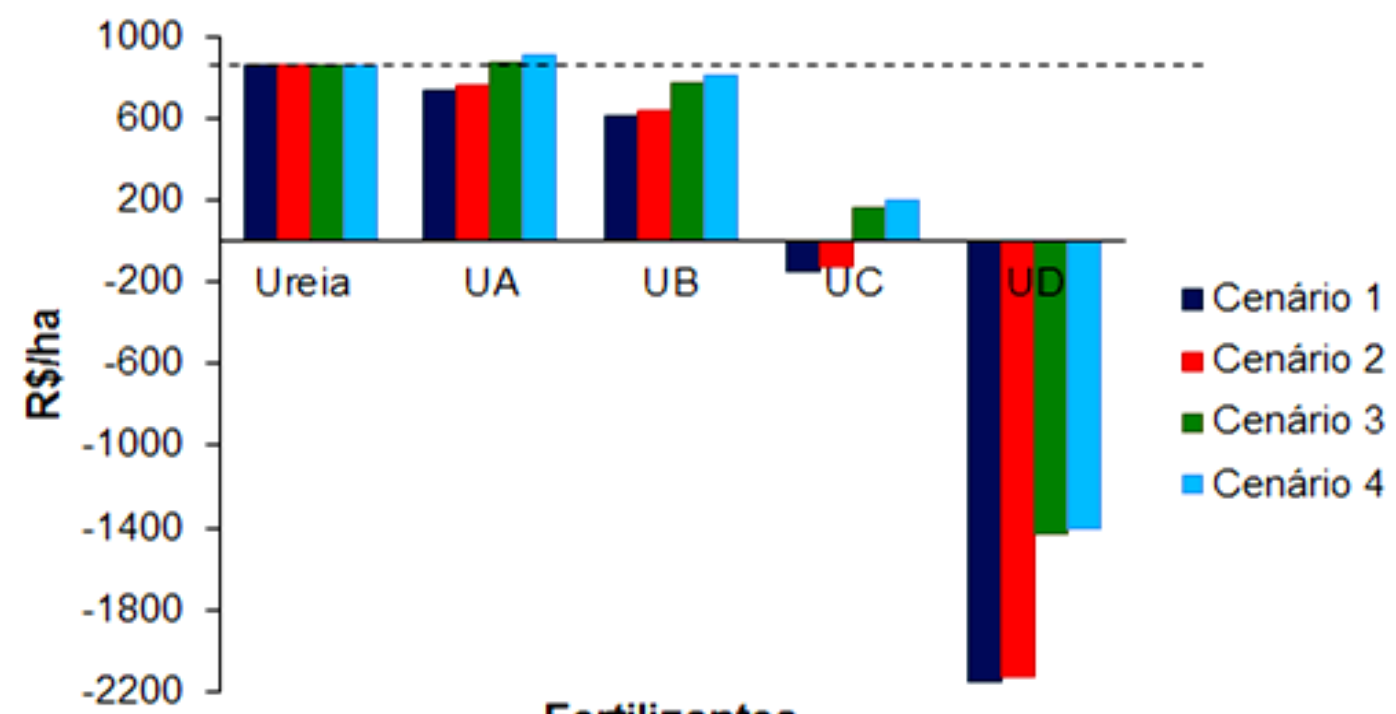

Figura 2. Comparativo dos retornos econômicos dos fertilizantes com e sem recobrimento nos cenários simulados

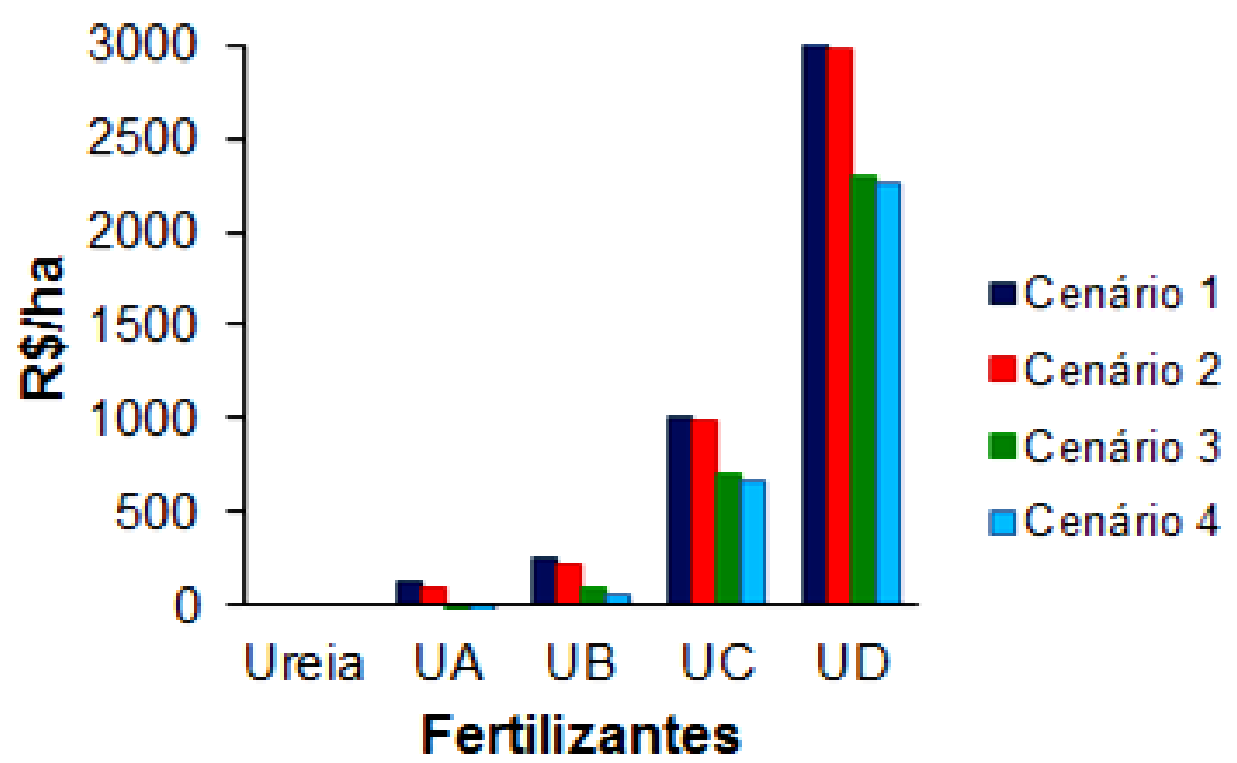

Figura 3. Comparativo dos aumentos nos custos de produção totais dos fertilizantes com e sem recobrimento nos cenários simulados 
Estes dados obtidos permitiram a verificação que, para que as alternativas (fertilizantes recobertos) fossem viáveis a aplicação no sistema de produção, via esta metodologia e considerando os cenários adotados, a redução de dose foi o fator que mais influenciou nos resultados e, a eliminação da operação de cobertura influenciou em menor intensidade a redução dos custos de produção.

Considerando esta redução de dose, é importante ressaltar que os trabalhos envolvendo estas tecnologias devem voltar-se ao aumento da eficiência dos fertilizantes no campo, desta forma o foco dos estudos para aumento de produtividade tornou-se fundamental. Dada esta consideração, calculou para todos os cenários a necessidade de aumento de produtividade que equalizou o uso da ureia convencional com as ureias recobertas.

Estes cálculos mostraram, para o cenário 1 e 2, necessidade de aumento para todos os produtos recobertos se estendendo desde 3,7 a $4,9 \mathrm{sc} / \mathrm{ha}(222-291 \mathrm{~kg} / \mathrm{ha})$ para o produto UA, aumentos de 8,3 a $9,5 \mathrm{sc} / \mathrm{ha}(500$ - $570 \mathrm{~kg} / \mathrm{ha})$ para UB e, para UC e UD onde os aumentos atingem valores elevados, os aumentos são de 36,8 a 37,9 sc/ha (2207 - 2276 kg/ha) e 111,6 a 112,8 sc/ha (6698 - 6767 kg/ha), respectivamente (Tabela 4).

Nos cenários 3 e 4, com exceção de UA onde o nivelamento com a ureia foi obtido com menores produtividades do que a considerada $(8,5 \mathrm{t} / \mathrm{ha})$, os produtos UB, UC e UD necessitam de 3,2, 26 e 85,8 sc/ha de aumento, respectivamente, no caso de duas operações de adubação e, 2,1, 24,8 e 84,7 sc/ha de aumento, respectivamente, considerando apenas uma aplicação de fertilizantes no cultivo. Salienta-se que mesmo nestes cenários as elevações de produtividades superiores a ureia convencional, necessárias, para UC e UD são elevadas podendo atingir 1560 a 5150 kg/ha, respectivamente (Figura 4). 

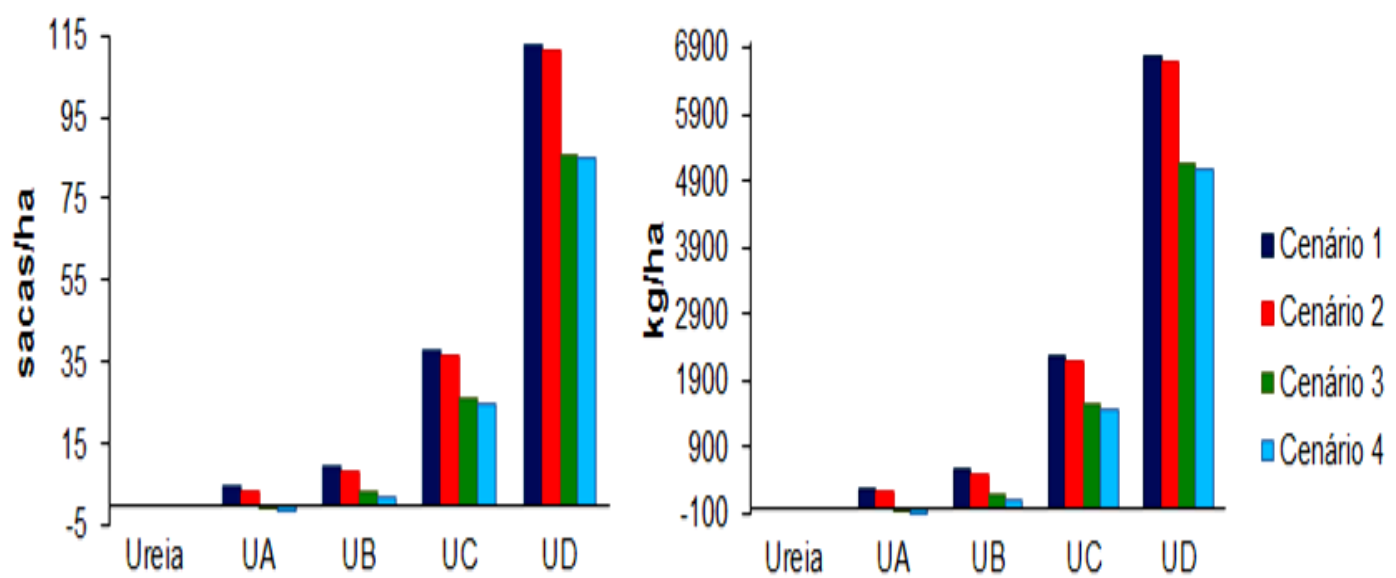

\section{Fertili zantes}

Figura 4. Necessidade de aumento de produtividade necessário ao ponto de nivelamento entre a ureia convencional e os produtos recobertos

\section{Considerações finais}

Os estudos envolvendo as análises agronômicas e econômicas necessitam ser desenvolvidas em conjunto por profissionais de ambas as áreas, multidisciplinarmente, para não somente constatar dados agronômicos do quanto as tecnologias empregadas podem ser viáveis, mas também informações econômicas, utilizando metodologias simples e úteis como a orçamentação parcial, que auxiliem o produtor na tomada de decisão.

Premissas de redução de doses aplicadas aos fertilizantes recobertos, que possibilitem redução dos custos de produção nos cultivos agrícolas necessitam não apenas de mais e muitos estudos detalhados, como também de maior divulgação de dados, assim a união de tais dados às análises de viabilidade econômica traçarão claramente as possibilidades e limitações das tecnologias e permitirão ao produtor uma escolha imparcial com base no desenvolvimento científico.

Desta forma, apenas com a colaboração clara, imparcial e direta das instituições privadas desenvolvedoras de produtos com as instituições científicas de ensino pesquisa e extensão, haverá a possibilidade do Brasil desenvolver todo seu potencial produtivo frente às necessidades da produção de alimentos, fibras e energia a um preço justo e viável ao produtor e a população.

\section{Conclusão}

Análise dos custos de produção da ureia aplicado no milho primeira safra no Estado de São Paulo e substituição desta pelos produtos recobertos, considerada dose 
de $180 \mathrm{~kg} / \mathrm{ha}$ de $\mathrm{N}$, aplicada em duas operações (plantio - $50 \mathrm{~kg} / \mathrm{ha}$; cobertura 130 $\mathrm{kg} / \mathrm{ha}$ ) (Cenário 1), os fertilizantes recobertos não são alternativas viáveis à substituição da ureia sem revestimento no sistema de produção. Estes produtos necessitam de aumentos de produtividade para tornarem-se viáveis gerando receitas adicionais que cubram os custos da aplicação de tecnologia ao fertilizante na forma convencional.

Os custos de produção das ureias recobertas considerando dose de $180 \mathrm{~kg} / \mathrm{ha}$ de $\mathrm{N}$ aplicada em apenas no plantio com ureia sem recobrimento mantida a realização de duas operações de adubação para milho (Cenário 2), os fertilizantes recobertos não são alternativas viáveis à substituição da ureia sem revestimento no sistema de produção. Estes produtos necessitam de aumentos de produtividade para tornarem-se viáveis gerando receitas adicionais que cubram os custos da aplicação de tecnologia ao fertilizante na forma convencional.

A eliminação de uma operação de adubação (cobertura) não é suficiente para viabilizar o uso dos fertilizantes recobertos frente à ureia sem recobrimento.

Considerado a dose de $180 \mathrm{~kg} / \mathrm{ha}$ de $\mathrm{N}$ na forma de ureia aplicada em duas operações (plantio - $50 \mathrm{~kg} / \mathrm{ha}$; cobertura $130 \mathrm{~kg} / \mathrm{ha}$ ) e para os produtos recobertos, redução de $20 \%$ da dose utilizada para ureia sem recobrimento (144 kg/ha de N), aplicada em duas operações (plantio $40 \mathrm{~kg} / \mathrm{ha}$; cobertura $104 \mathrm{~kg} / \mathrm{ha}$ ) em milho primeira safra (Cenário 3) a análise dos custos de produção do fertilizante recoberto (UA) é alternativa viável na substituição da ureia sem recobrimento no sistema de produção. Os demais fertilizantes (UB, UC e UD) não são viáveis e, para o serem, necessitam de aumentos de produtividade para assim cobrir os custos adicionais causados pela aplicação de tecnologia a ureia convencional.

Para a análise dos custos de produção das ureias recoberta na condição de redução de $20 \%$ da dose utilizada para ureia sem recobrimento (144 kg/ha), aplicada em uma operação de plantio para milho (Cenário 4), o fertilizante revestido (UA) é alternativa viável na substituição da ureia sem recobrimento no sistema de produção. Os demais fertilizantes (UB, UC e UD) não são viáveis e, para o serem, necessitam de aumentos de produtividade para assim cobrir os custos adicionais causados pela aplicação de tecnologia a ureia convencional.

A eliminação de uma operação de adubação (cobertura) potencializa os ganhos obtidos com o fertilizante UA e, para os demais, esta redução não foi suficiente para viabilizar o uso de UB, UC e UD em substituição a ureia convencional. 
Dessa forma, os aumentos na eficiência dos fertilizantes recobertos, que reflitam em aumentos de produtividade, podem viabilizar o uso destes insumos em substituição a ureia convencional.

\section{Referências}

AGRAFNP - Consultoria \& Comercio [AGRIANUAL]. 2014. Milho. In: Agrianual 2014: Anuário da agricultura brasileira. São Paulo, 2014. p. 349-378

Agência Nacional de Difusão de Adubos [ANDA]. 2011. Anuário Estatístico.

Blaylock, A. 2007. Novos fertilizantes nitrogenados: o futuro dos fertilizantes nitrogenados de liberação controlada. Piracicaba: IPNI. p. 8-10. (Informações Agronômicas, 120)

Companhia Nacional de Abastecimento [CONAB] 2014. Acompanhamento da safra brasileira - grãos, nono levantamento, v.1, n.9.

Fancelli, A.L.; Tsumanuma, G.M. 2007. Nitrogênio e enxofre nas culturas de milho e feijão In: Yamada, T.; Abdalla. S.R.S.; Vitti, G.C. (Ed) Nitrogênio e enxofre na agricultura brasileira. Piracicaba: IPNI Brasil. cap 13, p.445-486.

Fancelll, A.L. 2009. Nutrição e adubação do milho. In: Fancelli, A.L.;Dourado Neto,D. Milho manejo e produtividade. Piracicaba: USP/ESALQ/LPV. cap.5, p. 60-97

Hoffmann, R.; Engler, J. J. C.; Serrano, O.; Thame, A. C. M.; Neves, E. M. 1992. Administração da Empresa Agrícola, São Paulo: Pioneira. 325p.

Peoples, M.B.; Freney, J.R.; Mosier, A.R. 1995. Minimizing gaseous losses of nitrogen. In: Bacon, P.E. (Ed). Nitrogen fertilization in the environment. New York: Marcel Dekker, chap. 15, p.565-602.

Raij, B. van. 1991. Fertilidade do solo e adubação. Piracicaba: Ceres, 1991. 343 p.

Raij, B. Van; Cantarella, H.; Quaggio, J.A.; Furlani, A.M.C. 1997. Recomendações de adubação e calagem para o Estado de São Paulo. 2.ed. Campinas: Instituto Agronômico. 285p. (Boletim técnico, 100)

Shaviv, A. 2001. Advances in controlled-release fertilizers. Advances in Agronomy, San Diego, 71: 1-49.

. 2005. Controlled release fertilizers. In: IFA International Workshop On Enhanced-Efficiency Fertilizers, 2005, Frankfurt. Proceedings... Paris: International Fertilizer Industry Association, p. 1-15. 
Trenkel, M.E. 1997. Improving fertilizer use efficiency: controlled-release and stabilized fertilizers in agriculture. Paris: International Fertilizer Industry Association. $151 \mathrm{p}$.

Slow and controlled-release and stabilized fertilizers: an option for enhancing nutrient use efficiency in agriculture. Paris: International Fertilizer Industry Association, 2010. 167 p.

Vitti, G.C.; Heirinchs, R. 2007. Formas tradicionais e alternativas de obtenção e utilização do nitrogênio e do enxofre: Uma visão holística. In: Yamada, T.; Abdalla, S.R.S.; Vitti, G.C. (Ed.). Nitrogênio e enxofre na agricultura brasileira. Piracicaba: IPNI Brasil. cap. 4, p. 109-160. 